\title{
Popliteal Artery Aneurysms: Is Endovascular Reconstruction Durable?
}

\author{
DAVID ROSENTHAL, M.D., JOHN H. MATSUURA, M.D., and \\ MICHAEL D. CLARK, M.D.
}

\begin{abstract}
This initial series describes a minimally invasive, endovascular method of performing femoropopliteal in situ saphenous vein (SV) bypass and popliteal artery aneurysm (PAA) embolization. Twenty patients have undergone PAA operations. Eleven patients had conventional SV bypasses with proximal and distal ligation of the popliteal artery (PA), and nine patients underwent PAA embolization and an endovascular in situ SV bypass (EISB). The EISB bypass was performed using an angioscopic guided side branch occlusion coil system, whereas the PAAs were embolized with occlusion coils under fluoroscopic surveillance. After operation in the EISB group there were no deaths, no wound complications, and the mean hospital length of stay (LOS) was 2.1 days. Six operations were performed through two incisions, and three operations required an additional incision. Primary patency was $88 \%$ $(8 / 9)$, and all PAAs remained occluded by color flow ultrasonography at follow-up ranging from 4 to 23 months (mean 11.2 months). In the conventional bypass group there were no deaths, three patients $(27 \%)$ had wound complications, and the mean LOS was 6.2 days. Bypass patency at 42 months was $91 \%$ (10/11). This minimally invasive combined radiological and endovascular surgical technique obviates an extensive incision to harvest the SV and ligate the PAA proximally and distally. If long-term endovascular bypass graft patency and PAA occlusion rates prove to be similar to open operative results, the benefits of reduced wound complications, decreased hospital LOS, and increased health care savings support further investigation of this endovascular method for the treatment of PAA.
\end{abstract}

Keywords Endovascular treatment, popliteal artery aneurysms

Popliteal artery aneurysms (PAAs) are the most common peripheral aneurysms. If thrombosis or embolization from a PAA occurs, the limb loss rate, despite recent operative and thrombolytic advances, remains as high as

D.R., Clinical Professor of Surgery; J.H.M., Clinical Associate Professor of Surgery; M.D.C., Clinical Associate Professor of Surgery, Department of Vascular Surgery, Georgia Baptist Medical Center, Medical College of Georgia, Atlanta, GA.

Copyright (C) 2000 by Thieme Medical Publishers, Inc., 333 Seventh Avenue, New York, NY 10001, USA Tel. $+1(212)$ 584-4662. 0894-8046,p; 2000,12,2,55,62,ftx,en;pvs00063 
40\%. ${ }^{1}$ Appropriately, the PAA has been described as, "the sinister harbinger of sudden catastrophe." 2

Saphenous vein (SV) bypass with exclusion of the PAA remains the "gold standard" operation. Unfortunately, wound complications after SV bypass are common and have been reported to occur in up to one third of cases. ${ }^{3-5}$ The endovascular treatment of arterial aneurysms is being developed as an alternative method of treatment; however, only anecdotal information is currently available.6,7 A minimally invasive endovascular method of performing femoropopliteal in situ SV bypass with coil embolization of the PAAs has been developed, and the early experience is reported.

\section{MATERIALS AND METHODS}

Between 1990 and 199822 patients underwent elective operation for PAA. Any patient requiring urgent operation for limb salvage, with or without thrombolytic therapy, was excluded from this study. Twelve patients had "open" conventional SV bypass with exclusion of the PAAs by proximal and distal popliteal artery ligation; seven patients had reversed and five had in situ SV bypass. Ten patients underwent PAA embolization and an endovascular in situ SV bypass (EISB). All patients were men; ages ranged from 44 to 81 years (mean age 61 years). Comorbid conditions, which included hypertension, diabetes mellitus, cigarette smoking, and coronary artery heart disease, were similar between groups.

Prior to operation, all patients underwent color flow duplex ultrasonography to verify SV patency, size, and anatomic configuration. At EISB operation, standard incisions were made over the proximal femoral artery and at the location of the distal popliteal or tibial artery. The SV was dissected at each incision a sufficient length to permit anastomoses. After systemic heparin administration and division of the SV distally and from the common femoral vein, a retrograde valvulotome (Baxter Vascular Division, Irvine, CA) containing an irrigation channel for a 5\% dextrose-heparin $(1,000$ IU/L)-papaverine $(30 \mathrm{mg} / \mathrm{L})$ solution was introduced into the distal saphenous vein; the side branch occlusion system (Baxter Vascular Division) containing a movable angioscope and occlusion catheter was introduced into the proximal SV; its component parts are a movable angioscope and the introducer catheter, within which is the coil delivery catheter, which in turn contains a preloaded Gianturco occlusion coil (Target Therapeutic, San Jose, CA) and guide wire (Fig. 1). ${ }^{7}$

Prior to valvulotomy, the angioscope is placed in the "extended position," which allows visualization of the vein lumen in a 360-degree, circumferential fashion. The valvulotome cutting blades at this time are retracted to minimize any potential for intimal trauma. When a set of valve leaflets is identified, the cutting blade is extended and the valve leaflet is engaged (Fig. 2). With gentle distal traction on the valvulotome, vavulotomy of each valve 


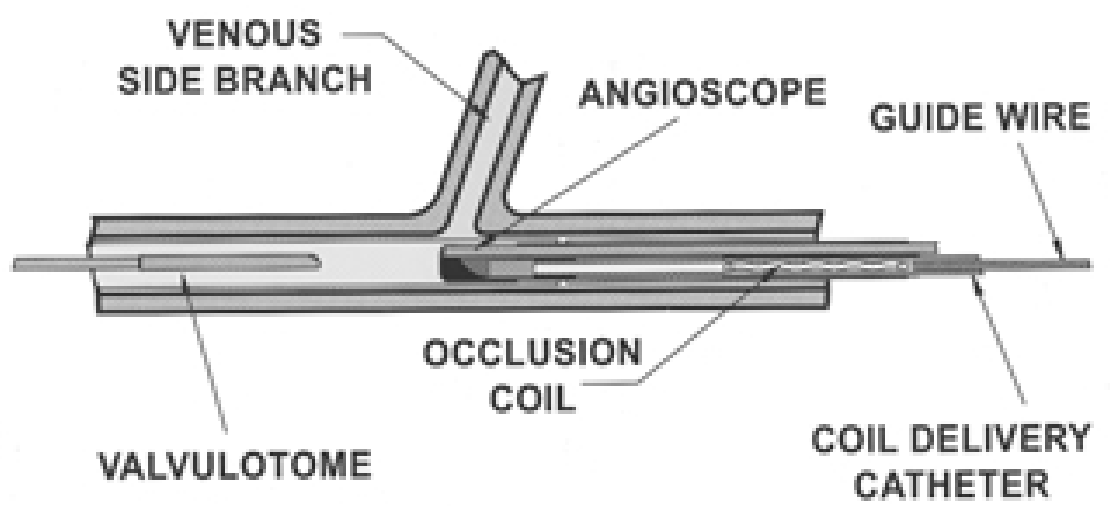

Fig. 1 Retrograde valvulotome and side branch occlusion system. (From Rosenthal D. Angioscopy in vascular surgery. Cardiovasc Surg; 1997;245-255. With permission from Elsevier Science.)

leaflet under direct angioscopic surveillance is performed. This angioscopic guided technique ensures safety and completeness of valvulotomy.

Most major side branches are located adjacent to the site of the vein valves; therefore, side branch occlusion can be performed either before or after valvulotomy at the surgeon's discretion. During side branch occlusion, the angioscope is placed into the "retracted position," which better allows the operator to visualize the side branch orifice. The coil delivery catheter, which contains the preloaded occlusion coil and guide wire, is then guided into the side branch orifice. The guide wire is advance, a coil is deployed (Fig. 3 ) into the venous side branch, and the side branch is embolized (Fig. 4). With this new angioscopic guided system, valvulotomy and side branch occlusion may by easily and safely performed. The cost of the valvulotome, side branch occlusion system, and occlusion coils is approximately $\$ 2000$.

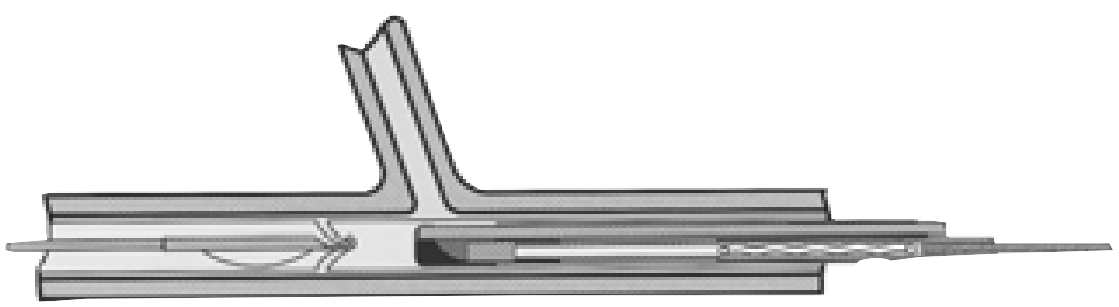

\section{VALVE LEAFLET \\ ENGAGED}

Fig. 2 Under angioscopic surveillance, the valvulotome cutting blade is extended and the valve leaflet engaged. (From Rosenthal D. Angioscopy in vascular surgery. Cardiovasc Surg; 1997;245-255. With permission from Elsevier Science.) 


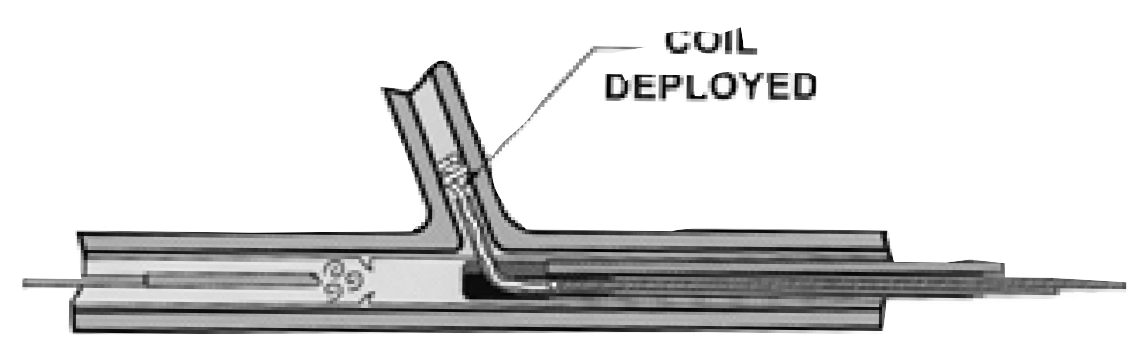

Fig. 3 The coil deliver catheter containing an occlusion coil and guide wire is guided into the side branch. (From Rosenthal D. Angioscopy in vascular surgery, 1997;5:245-255. Used with permission from Elsevier Science.)

The popliteal artery distal to the PAA was then divided and the proximal end oversewn. A common femoral arteriotomy was made, and a 7-French introducer sheath was placed into the superficial femoral artery over a 0.035inch guide wire. Under fluoroscopic surveillance the guide wire was passed to the level of the PAA, which was thrombosed with 8- and 10-mm Gianturco occlusion coils (Fig. 5). On average, four Gianturco coils were required to occlude the PAAs. Following embolization of the PAA, the proximal and distal anastomoses were completed in a standard fashion. All distal anastomoses were to the distal popliteal artery or tibioperoneal segment, thus avoiding an additional incision. ${ }^{8,9}$

Completion arteriograms were performed to verify PAA thrombosis and to identify any missed or nonoccluded arterial venous fistulas in the vein graft or technical imperfections and to assure patency of the outflow anastomosis (Fig. 6). Similarly, all patients who underwent conventional reversed or in situ SV bypass and PAA exclusion had completion arteriograms. All patients were followed with serial color flow ultrasonography 3, 6, and 12 months after operation and biannually thereafter.

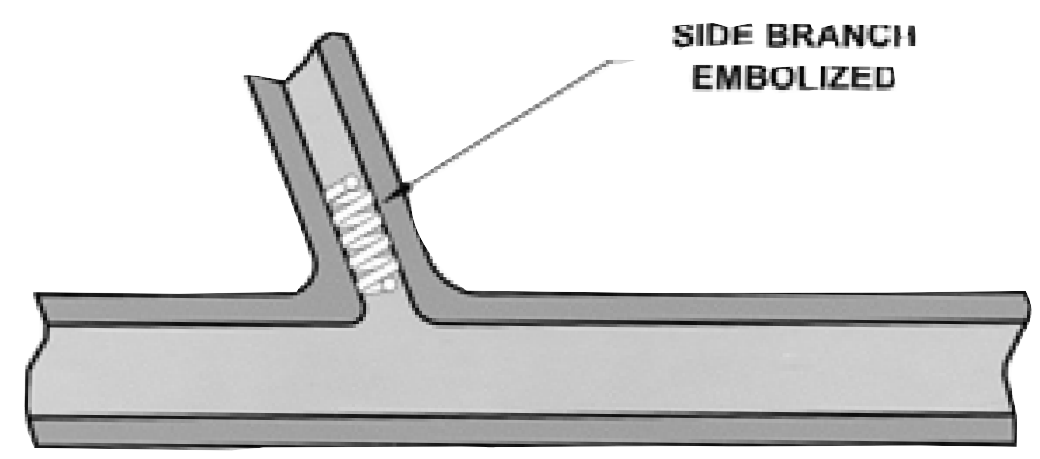

Fig. 4 The coil is deployed and the side branch is embolized. 


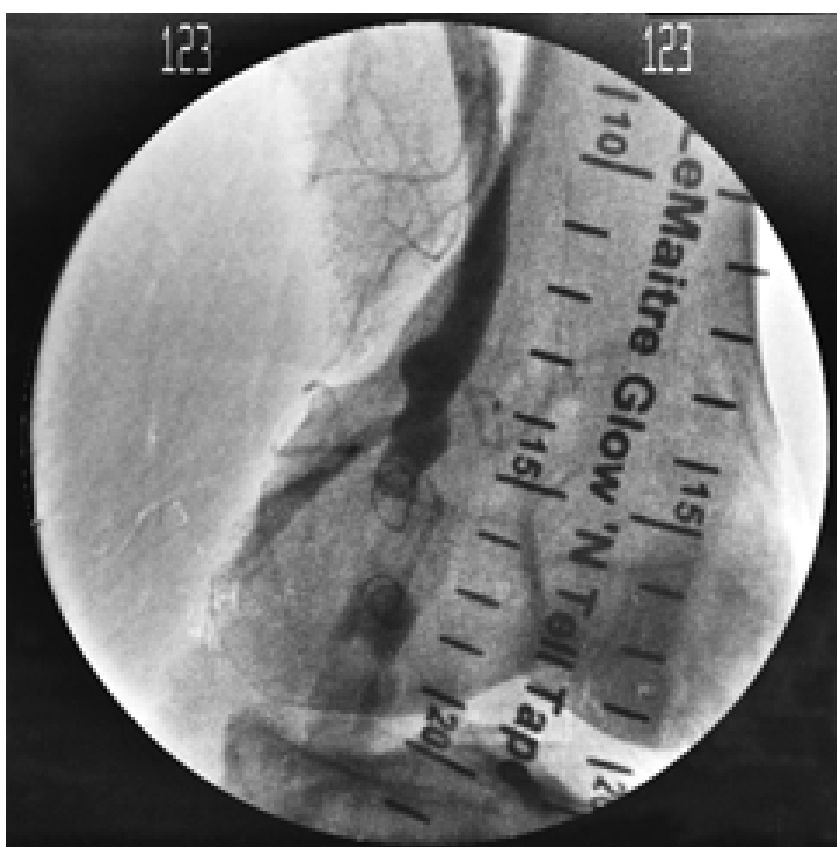

Fig. 5 Coil occluded popliteal artery aneurysm.

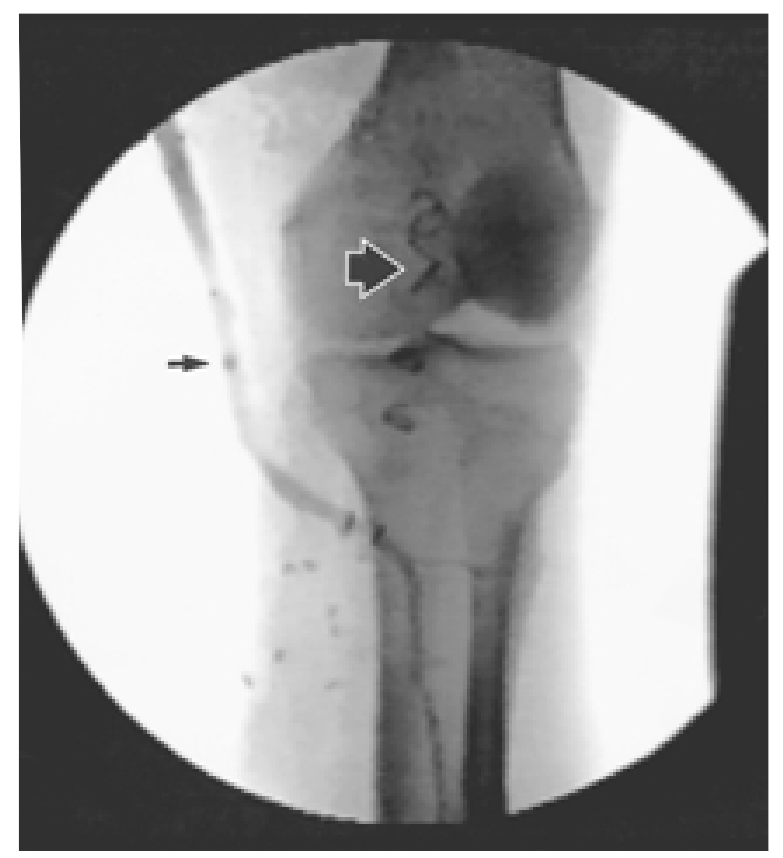

Fig. 6 Completion arteriogram demonstrating coil occluded popliteal artery aneurysm (large arrow) and patent endovascular femoropopliteal in situ saphenous vein bypass (small arrow). 


\section{RESULTS}

The mean length of EISB operation was 2.75 hours with a mean blood loss of $180 \mathrm{ml}$. After operation in the EISB group there were no deaths or wound complications, and the mean hospital length of stay (LOS) was 2.1 days. Six operations were performed through two incisions, whereas three operations required an extra incision to ligate large side branches $(>5 \mathrm{~mm})$ or missed arterial venous fistulae. No "late" arterial venous fistulae developed during follow-up. Primary bypass patency was $90 \%(9 / 10)$, and all PAAs remained occluded by color flow ultrasonography at follow-up ranging from 4 to 23 months (mean 13.6 months). The one graft occlusion occurred at 3 months follow-up, and the patient refused reoperation. In the reversed or in situ SV bypass and PAA exclusion group, there were no deaths, three patients $(25 \%)$ had wound complications, and the mean LOS was 6.2 days. The mean length of operation was 2.3 hours with an estimated blood loss of $230 \mathrm{ml}$. Bypass patency at 42 months was $92 \%(11 / 12)$.

\section{DISCUSSION}

PAAs historically are found in older males, are often bilateral, and are frequently associated with other arterial aneurysms. Elective repair of an asymptomatic PAA is recommended because of the significant morbidity associated with an acute thromboembolic event.10,11 The "gold standard" operation for PAA is femoropopliteal-tibial SV bypass with exclusion (proximal and distal ligation) of the aneurysm to prevent expansion and/or distal embolization. ${ }^{12,13}$ Wound complications after femoropopliteal bypass for occlusive disease, however, with SV are common and have been reported in up to one third of cases. ${ }^{3-5}$ These wound complication are usually due to ischemia of the posterior flap along the thigh incision, which may cause cellulitis, lymphangitis, and tissue necrosis. In this series, $25 \%$ of patients undergoing conventional vein bypass with PAA exclusion had a wound complication. Among the EISB patients, no wound complications occurred as the endovascular method of performing femoropopliteal in situ SV bypass and PAA embolization avoided a long incision in the thigh to expose the SV for harvest and/or to ligate the proximal popliteal artery or an incision across the knee joint to mid calf to expose SV at this location. ${ }^{10}$

In this healthcare cost-conscious era, a concern that must be addressed is the cost-effectiveness of this endovascular technique. 1996 Healthcare Finance Administration data on Diagnosis Related Group payments for 4295 patients undergoing popliteal aneurysm resection (ICD 9 CM 442.3) demonstrated a mean hospital LOS of 6.2 days and mean loss to the hospital of $\$ 7104$ per patient, or loss of over $\$ 30,500,000$ in $1996 !^{11}$ By avoiding a long leg incision and the potential associated wound com- 
plications, which prolong the hospital LOS, any additional cost of the endovascular instrumentation (\$2000) may be readily offset. Indeed, the mean hospital LOS for the EISB patients was 2.1 days versus 6.2 days for the conventional SV bypass group. It appears that the EISB and PAA embolization technique will not only benefit the patient with a less invasive procedure but it may remedy the high cost to hospitals of caring for patients with PAAs as well.

Endovascular stent grafts are currently under investigation for the treatment of abdominal aortic aneurysm, and this technology may prove valuable and efficacious for PAA. Case reports utilizing a variety of flexible stent grafts via a percutaneous approach have demonstrated feasibility for the treatment of PAA; however, only short-term follow-up (less than 1 year) is available. ${ }^{6,7}$ A major concern regarding the use of popliteal endoluminal stent grafts is possible extrinsic compression and deformation of the stent from muscle and tendons due to flexion of the knee joint. Most PAAs extend across the knee joint, and the dynamic flexion and extension forces exerted across the joint may compromise the long-term patency of endoluminal stent grafts. This, however, remains to be evaluated.

As this was a small series of patients, further studies will determine whether this minimally invasive EISB bypass and PAA embolization technique yields long-term patency rates similar to the conventional open bypass and PAA exclusion operation. Potentially, however, the benefits of reduced wound complications, shortened hospital stay and recuperation, and decreased healthcare costs make this endovascular method for the treatment of PAA intriguing.

\section{REFERENCES}

1. Vermilion BD, Kimmins SA, Pace WG, et al. A review of one hundred forty seven popliteal aneurysms with long-terms follow-up. Surgery 1981;90:1009-1014

2. Gifford RW Jr, Hines EA, Janes JM. An analysis and follow-up study of one hundred popliteal aneurysms. Surgery 1953;33:284-293

3. Schwartz ME, Harrington EB, Schanzer H. Wound complications after in situ bypass. J Vasc Surg 1988;7:802-807

4. Utley JR, Thomason ME, Wallace JD, et al. Preoperative correlates of impaired wound healing after saphenous vein excision. J Thorac Cardiovasc Surg 1989;98:147-149

5. Wengrovitz M, Atnig RG, Gifford RM, et al. Wound complications of autogenous subcutaneous infrainguinal arterial bypass surgery: Predisposing factors and management. J Vasc Surg 1990;11:156-163

6. Kudelko PE, Alfaro-Franco C, Dietrich EB, Krajcer Z. Succesful endoluminal repair of a popliteal artery aneurysm using the Wall graft endo prosthesis. J Endovasc Surg 1998; 5:373-377

7. Burger T, Meyer, F, Tauterhahn J, et al. Initial experience with percutaneous endovascular repair of popliteal artery lesions using a new PTFG stent-graft. J Endovasc Surg $1998 ; 5: 365-372$

8. Rosenthal D, Atkins CP, Shuler FW, et al. Popliteal artery aneurysm treated with a minimally invasive endovascular approach. J Vasc Surg 1998;5;60-64 
9. Rosenthal, D, Dickson C, Rodriquez FJ, et al. Infrainguinal endovascular in situ saphenous vein bypass: Ongoing results. J Vasc Surg 1994;20:389-395

10. Carpenter JP, Maker CF, Roberts B, et al. Popliteal artery aneurysms: Current management and outcome. J Vasc Surg 1994;19:65-73

11. Anton GE, Hertzer NR, Bevin EG, et al. Surgical management of popliteal aneurysms. J Vasc Surg 1986;3:125-134

12. Whitehouse WM Jr, Wakefield TW, Graham LM, et al. Limb-threatening potential of arteriosclerotic popliteal artery aneurysms. Surgery 1983;93:694-699

13. HealthCare Finance Administration. Diagnostic codes for all short-stay hospitals. 1996 
Most popliteal artery aneurysms (PPAs) are repaired with a relatively short reversed saphenous vein (SV) bypass graft from the distal superficial femoral or proximal popliteal artery to the distal popliteal artery or to the tibioperoneal trunk. This bypass is easily constructed through a medial approach, with an incision starting in the mid to distal thigh and ending in the proximal calf. The SV can be harvested through the same incision used for arterial exposure. A skin bridge may be left at the knee resulting in two separate incisions, if this is the surgeon's preference. This operative approach avoids a groin incision and the associated wound problems, facilitates proximal and distal ligation of the aneurysm, and results in the shortest possible bypass graft. This is a wellestablished procedure, with a proven record of success and durability. In our experience, these relatively short bypasses have few wound problems and do not require a prolonged hospitalization. This is particularly true in patients without symptoms of ischemia, undergoing an elective reconstruction.

In the current series, endovascular in situ SV bypass is compared with traditional SV bypass for the elective treatment of PAA. Patients undergoing the endovascular procedure had fewer wound complications and a shorter length of hospital stay compared with those undergoing the traditional procedure. These benefits are presumed to be the result of the less-extensive incisions required for the endovascular procedure compared with those required the traditional procedure. All endovascular procedures were common femoral to below knee popliteal bypasses. The anatomic details of the traditional bypass procedures were not described, but one must assume that common femoral to below knee popliteal bypasses were performed.

The procedure described by Dr. Rosenthal and colleagues is not truly an endovascular reconstruction but rather PAA embolization followed by an angioscopic assisted in situ SV bypass. The vascular reconstruction is performed using an in situ SV graft and standard vascular anastomotic techniques. Angioscopic side branch occlusion is used to minimize or eliminate the incisions required in preparation of the vein graft. This is a developing technology, which has the benefit of limiting the extent of the incisions needed in patients undergoing in situ SV bypass grafting.

Although this series demonstrates the technical feasibility of the procedure along with acceptable short-term success, we are not convinced that this is the correct procedure for the treatment of PAAs. The patients in this series all

M.E.M., Assistant Professor of Surgery, Division of Vascular Surgery, University of Pennsylvania School of Medicine; J.P.C., Associate Professor of Surgery, Division of Vascular Surgery, University of Pennsylvania School of Medicine, Philadelphia, PA.

Copyright (C) 2000 by Thieme Medical Publishers, Inc., 333 Seventh Avenue, New York, NY 10001, USA. Tel. +1(212) 584-4662. 0894-8046,p; 2000,12,2,63,64,ftx,en;pvs00064 
had patent superficial femoral arteries that were accessed for embolization of the PAA. Presumably these patients would have been candidates for the standard bypass procedure originating from the distal superficial femoral artery or the proximal popliteal artery. This would have eliminated the need for the harvesting of a long segment of SV, and the groin incision would be replaced with a distal thigh incision. It is unlikely that there is a significant difference in morbidity between a procedure with a groin incision and a procedure with a distal thigh incision.

Angioscopic assisted in situ SV bypass is a technique that has the potential for reducing the wound complications frequently seen with long distal bypass procedures performed for severe ischemia. Patients with PAAs undergoing elective surgery are usually reconstructed with a relatively short bypass graft with very little morbidity. The procedure described by the authors would be useful in treating patients with superficial femoral artery occlusive disease in conjunction with a PAA. These patients require a long bypass graft and may benefit from this technique. 
David Rosenthal, M.D.

Drs. Mitchell and Carpenter raised several interesting points in their "expert commentary." They are correct in surmising that patients in this series had patent superficial femoral arteries that allowed embolization of the popliteal artery aneurysms (PAAs), and certainly these patients might have undergone a standard "open" bypass from the distal femoral artery.

It was of interest to note that Drs. Mitchell and Carpenter advocated harvesting saphenous vein (SV) from the "distal thigh and ending in the calf." This, however, is contrary to the technique used by most vascular surgeons where the SV is harvested from the thigh because of its larger size. Wound complications after SV harvest are common, morbid, and expensive. By avoiding a long SV harvest incision and its associated expensive wound complication potential, we benefit not only the patient but the hospital as well.

The endovascular in situ SV bypass (EISB) and PAA embolization procedure is not for every patient. Size and quality of the SV are limiting factors, and in smaller veins, those less than $3.5 \mathrm{~mm}$ in diameter, the side branch occlusion technique is not appropriate as intimal trauma may cause vein graft failure. It is of interest to note, however, that in the Albany group's landmark report on 2058 in situ SV bypasses, the "length" of the vein bypass did not adversely effect long-term patency. ${ }^{1}$ Hopefully, the longer EISB grafts in our patients perform as well as short-segment grafts advocated by Drs. Mitchell and Carpenter.

Our series of patients is small and requires long-term follow-up to evaluate the merit of this procedure. Nevertheless, the EISB and PAA technique is a safe, minimally invasive, and cost-effective operation in appropriate patients.

\section{REFERENCES}

1. Shah DM, Darling RC, Chang BB, Leather RP. Long-term results of in situ saphenous vein bypass: Analysis of 2058 cases. Ann Surg 1995;4:438-448. 
